\title{
Locally Advanced Carcinoma of Maxillary Sinus with Orbital Involvement
}

\author{
Gaurav Mittal ${ }^{1} \quad$ Mudit Agarwal $^{2} \quad$ Anmol Agarwal ${ }^{1}$ \\ ${ }^{1}$ Department of Oral and Maxillofacial Surgery, Institute of Dental \\ Studies and Technologies, Ghaziabad, Uttar Pradesh, India \\ ${ }^{2}$ Head and Neck Surgical Oncology, Rajiv Gandhi Cancer Institute \\ and Research Centre, New Delhi, India
}

\section{Ritesh Garg ${ }^{1}$ Abhishek Rathi ${ }^{1}$ Pratiksha Dwivedi ${ }^{1}$}

Asian J Oncol:2021;7:8-12

\begin{abstract}
Address for correspondence Gaurav Mittal, BDS, MDS, Department of Oral and Maxillofacial Surgery, Institute of Dental Studies and Technologies, Modinagar 201204, Uttar Pradesh, India (e-mail: drgauravmittal@rediffmail.com).
\end{abstract}

\author{
Abstract \\ Keywords \\ - maxillary sinus \\ carcinoma \\ - en bloc radical \\ maxillectomy \\ - orbital exenteration \\ - anterolateral thigh flap \\ - adjuvant therapy
}

Maxillary sinus carcinoma is a rare neoplasm, with incidence of 0.2 to $0.8 \%$ of all systemic malignancies. Patients are diagnosed in the advanced stage when there is orbital invasion with abnormal ocular signs and symptoms. The standard treatment of maxillary sinus carcinoma is en bloc radical maxillectomy along with orbital exenteration, followed by postoperative adjuvant therapy. Orbital exenteration is used when the malignancy extends to the orbit in case of squamous/basal cell carcinoma. The aim of the current review is to provide an insight into the management of advanced maxillary carcinoma with orbital involvement in a 42-year-old male.

\section{Introduction}

Sinonasal malignancies involve maxillary, ethmoid, frontal, sphenoid and nasal cavities, of which maxillary antrum is 25 to $60 \%$ involved and sphenoid sinus is the least commonly involved. Sinonasal tumors constitute 0.2 to $0.8 \%$ of all systemic malignancies and 3 to $4 \%$ of all head and neck neoplasms. These are more commonly seen in elderly male patients. Local recurrences occur in approximately 25 to $35 \%$ of cases and distant metastases in $10 \%$ of cases. Sinonasal tumors have relatively poor prognosis, because they are diagnosed at advanced stages of disease due to relative paucity of pain in the early stages, with other clinical symptoms such as nasal congestion, rhinorrhea, epistaxis, and sinus pressure being treated for presumed infection. ${ }^{1,2}$

Squamous cell carcinoma is the commonest type of sinonasal malignancy that has been reported mostly in people who come into contact with hazardous material such as wood furniture, isopropyl alcohol, radium, nickel, chromium pigment, bantu snuff, thorotrast, mustard gas, polycyclic hydrocarbons, and cigarettes.,., Invasion of malignant paranasal and nasal sinus neoplasms into the surrounding structures and beyond the sinuses occur in $75 \%$ of cases and up to the orbit

published online July 17,2020
DOI https://doi.org/ 10.1055/s-0040-1714308 ISSN 2454-6798. in $45 \%$ of cases. The orbital invasion may exhibit proptosis, facial or eye pain, visual loss, epiphora, globe displacement, limitation of extraocular movements, diplopia, inequality of palpebral fissures, conjunctival chemosis, a palpable mass within the orbit, bony erosion, fundus findings (optic disc edema), and dacryocystitis. ${ }^{5-7}$

The treatment of sinonasal malignancies requires a multidisciplinary team approach that includes radiologists, head and neck surgeons, neurosurgeons, oral prosthetics specialists, radiation oncologists, and medical oncologists. The treatment of choice for sinonasal malignancies includes combined surgery and irradiation and more concern about oncologic cure. The advent of new diagnostic technologies, advances in anesthesia, perioperative antibiotic prophylaxis, surgical reconstruction, and adjunctive therapy have prompted oncologic surgeons to become more conservative regarding the extirpation of functional structures. ${ }^{8}$

\section{Case Report}

A 42-year-old male presented with complaints of gradually increasing painless swelling over the left side of the face since 3 to 4 months with associated focal pain. Physical 
examination revealed thin built with no history of systemic illness. No history of trauma was present. On examination, there was a well-defined irregular fungating growth approximately $6 \times 8 \mathrm{~cm}$, involving left maxilla infiltrating into the left orbit, with displacement of left eyeball laterally and superiorly. On extraoral examination, the lesion extended from lateral border of nose to cheek bone transversely and vertically from nasolabial fold to supraorbital margin. Left eyeball was not seen due to growth. The surrounding area of lesion was glossy, red inflamed, and edematous (-Fig. 1).

The inflamed lesion was tender on palpation. Mouth opening was normal. Left submandibular lymph nodes of $3 \times 3 \mathrm{~cm}$ were palpable with stony hard consistency and fixed to the neighboring structures with clinical extra capsular extension. Level II and III nodes were also enlarged. Left-sided malar swelling and nasal block were present. Left hemipalate was involved with the tumor. There were no significant findings from other systemic examinations.

Contrast-enhanced CT of orbit and paranasal sinuses (PNS) was suggestive of a large, well-defined heterogeneously hyperdense solid exophytic mass lesion seen involved with left maxillary sinus and extensive destruction of its walls. The mass is seen extending into the left orbit and displacing the globe superolaterally with loss of fat plane ( - Fig. 2). Medially, the mass is seen involved with left osteomeatal complex, turbinates, ethmoid air cells, and lateral nasal wall and septum ( - Fig. 3).

Routine investigation for preanesthetic clearance was carried out, and counseling of the patient in the presence

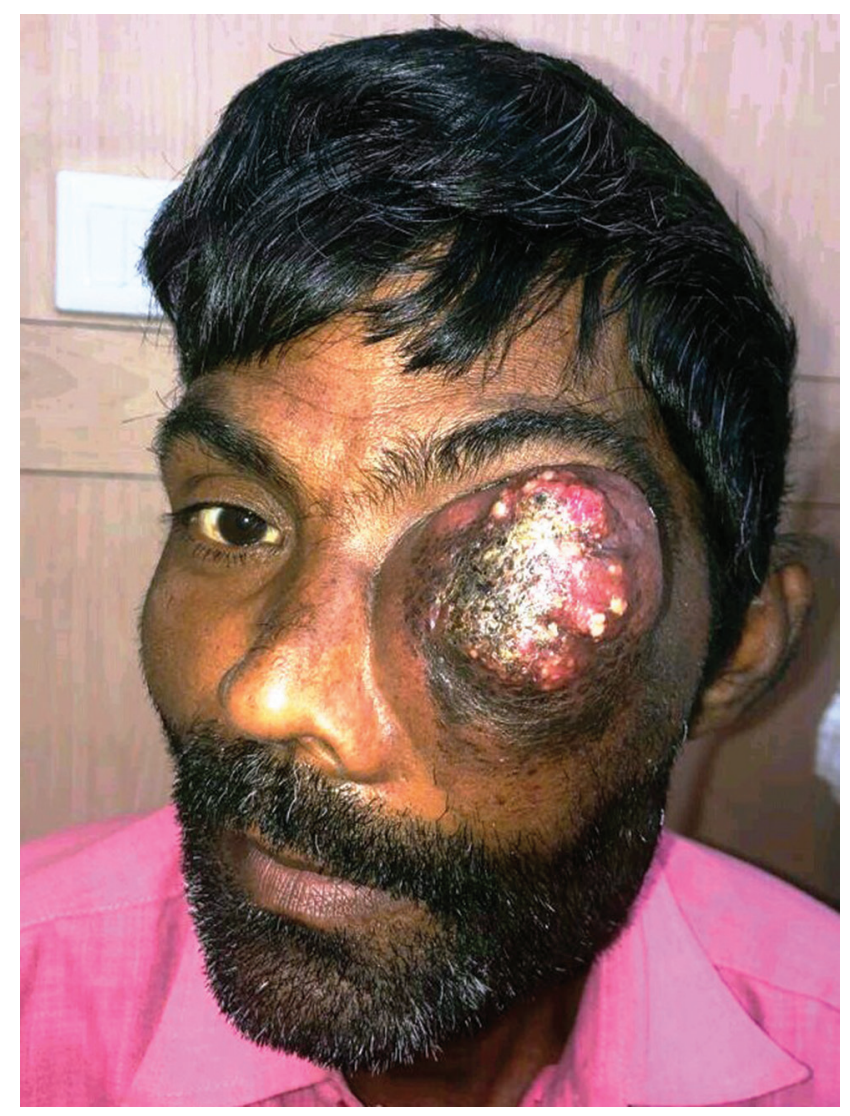

Fig.1 Preoperative extraoral image. of guardians was conducted, explaining the risks and benefits of surgery. Written informed consent for surgery and removal of one eye was obtained after counseling. Left radical Type IIIb maxillectomy, according to Cordeiro's classification, with orbital exenteration, followed by left modified radical neck dissection type III, reconstruction with left anterolateral thigh flap and, finally, adjuvant therapy were planned.

Tracheostomy was performed and general anesthesia was administered through the tracheal tube. The patient was positioned supine with head up, and parts were cleaned and draped. The Weber-Fergusson incision was made around the involved

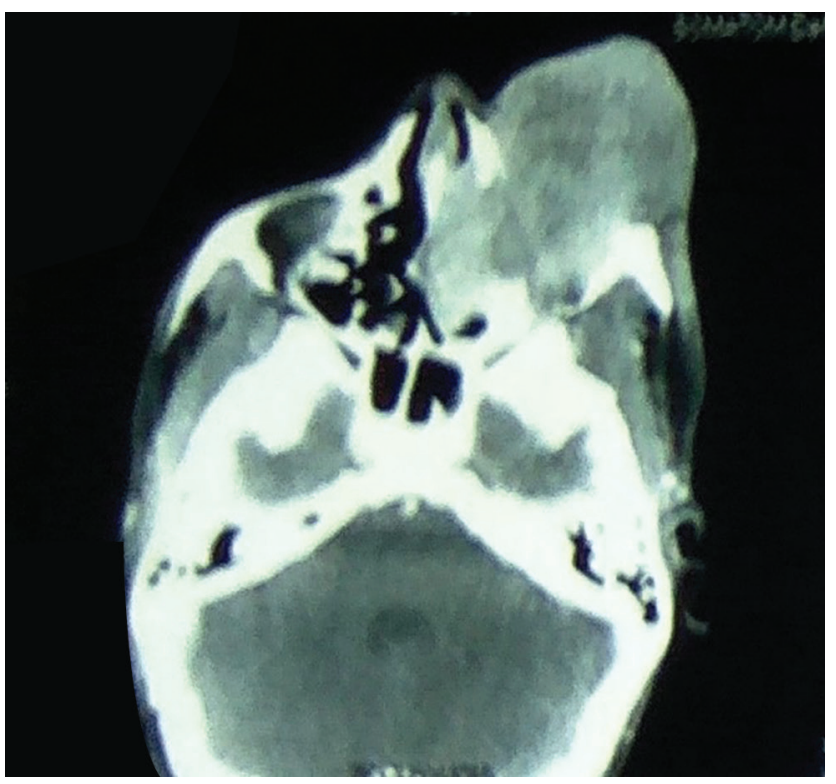

Fig. 2 CECT PNS (contrast-enhanced computed tomography and paranasal sinuses): axial View depicting lesion extending into left orbit and displacing the globe superolaterally with loss of fat plane.

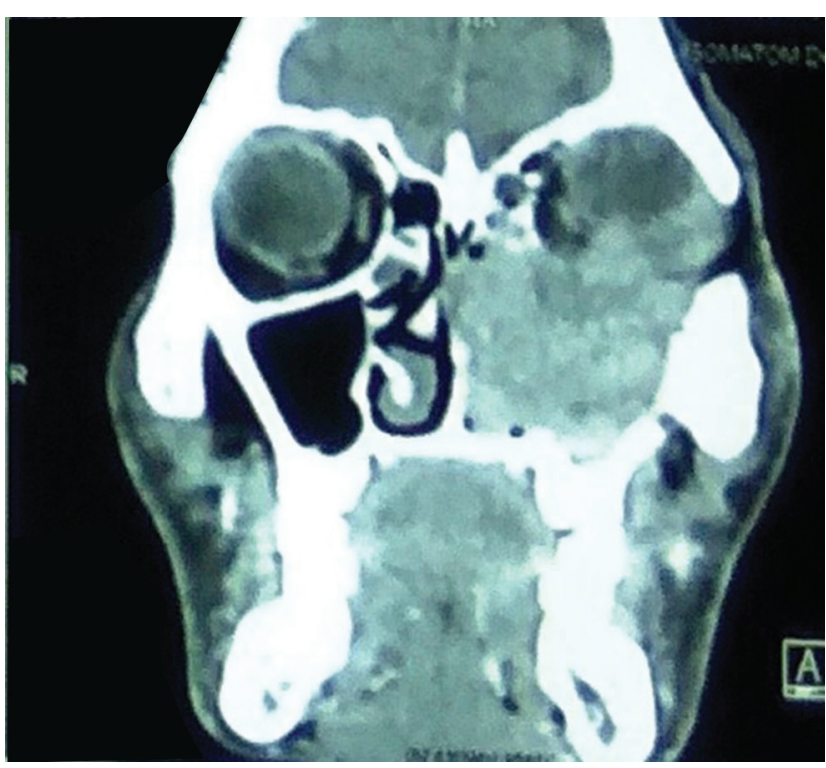

Fig. 3 CECT PNS (contrast-enhanced computed tomography and paranasal sinuses): corornal view showing the mass present medially involving left osteomeatal complex, turbinates, ethmoid air cells and lateral nasal wall and septum. 
skin. The left cheek flap was raised and radical maxillectomy was performed, along with upper gingivobuccal sulcus and an buccal mucosa resection done as an en bloc specimen, by giving anterior cut through extraction socket of left upper central incisor, midline palate cut, posterior cut at pterygoids, superior cut at midline incorporating the left nasal bone, inferiorly midline cut along nasal floor incorporating the lateral nasal wall, sparing the left ala of nose, and lateral cut through lateral orbital rim and zygomatic arch. The orbital cut was placed along the medial orbital wall, incorporating the floor and orbital contents and laterally connected to the cut placed on the lateral orbital wall. The specimen was oriented and sent for frozen section (-Fig.4). Coronoidectomy was done for access. Hemostasis was achieved. Modified radical neck dissection (type III) was performed at an extended neck position turned toward right. The skin flap was elevated in subplatysmal plane (-Fig. 5).

After maxillectomy, the defect (-Fig. 6) was closed by using anterolateral thigh flap with double paddle of $25 \times 10 \mathrm{~cm}$ for intraoral reconstruction of hard palate and buccal mucosa, extraoral reconstruction of orbit, maxilla and zygoma, muscle to fill the cavity, and a portion of the flap turned into form the lateral wall of the nose. The left thigh was used as a donor site with three musculocutaneous perforators, including fascia and partial vastus lateralis muscle ( - Fig. 7 ). The donor site was closed primarily over room suction drain, and distal pulsation of femoral artery was confirmed. Anastomosis of facial artery was done by using 9-0 nylon, and that of common facial vein was done by 3-0 coupler. The flap was evaluated at the end of the procedure and found to be well-perfused (-Figs. 8).

\section{Discussion}

Cancer of the maxillary sinus, a lethal neoplasm with an insidious onset, is most often diagnosed at an advanced stage

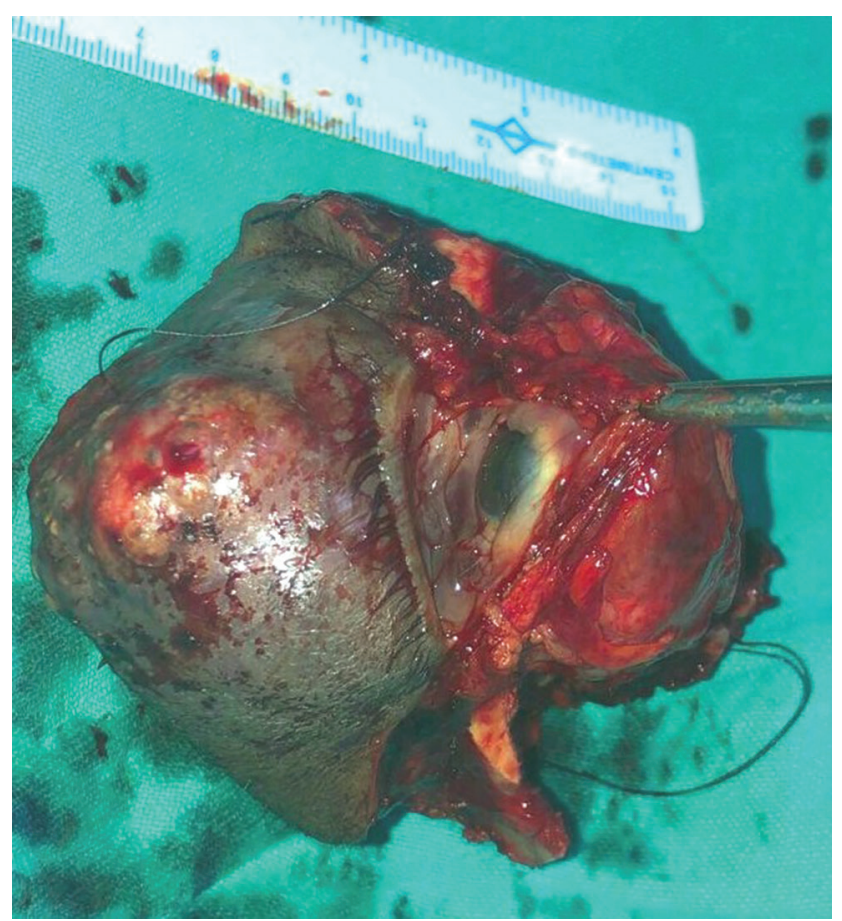

Fig. 4 Labeled excised specimen. of the disease, because it might be misdiagnosed with common sinus infection at the early stage of clinical symptoms. It is most commonly predilected in elder males. Clinical classification of the cancer is dependent on the location of antrum involvement as well as its size. ${ }^{9}$

Staging systems are used to define the extent of neoplastic disease and provide some basis to determine prognosis. The tumor, node, and metastases (TNM) system of classification of maxillary sinus cancers is based on an imaginary line, separating the maxillary antrum into anteroinferior and superoposterior compartments, as shown by Ohngren ${ }^{10}$ in 1933. Lederman ${ }^{11}$ drew two parallel lines through the antral

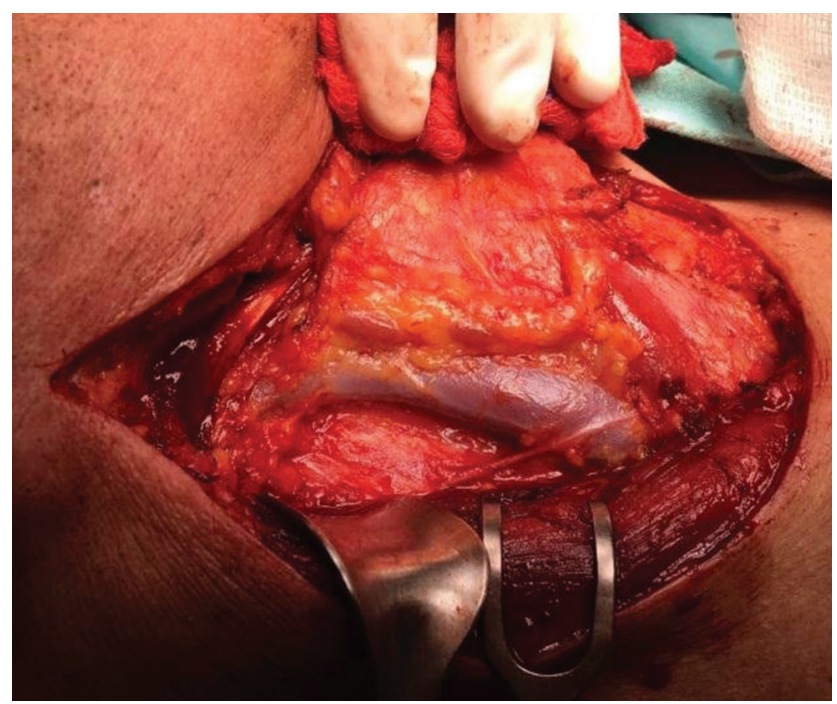

Fig. 5 Modified radical neck dissection type III.

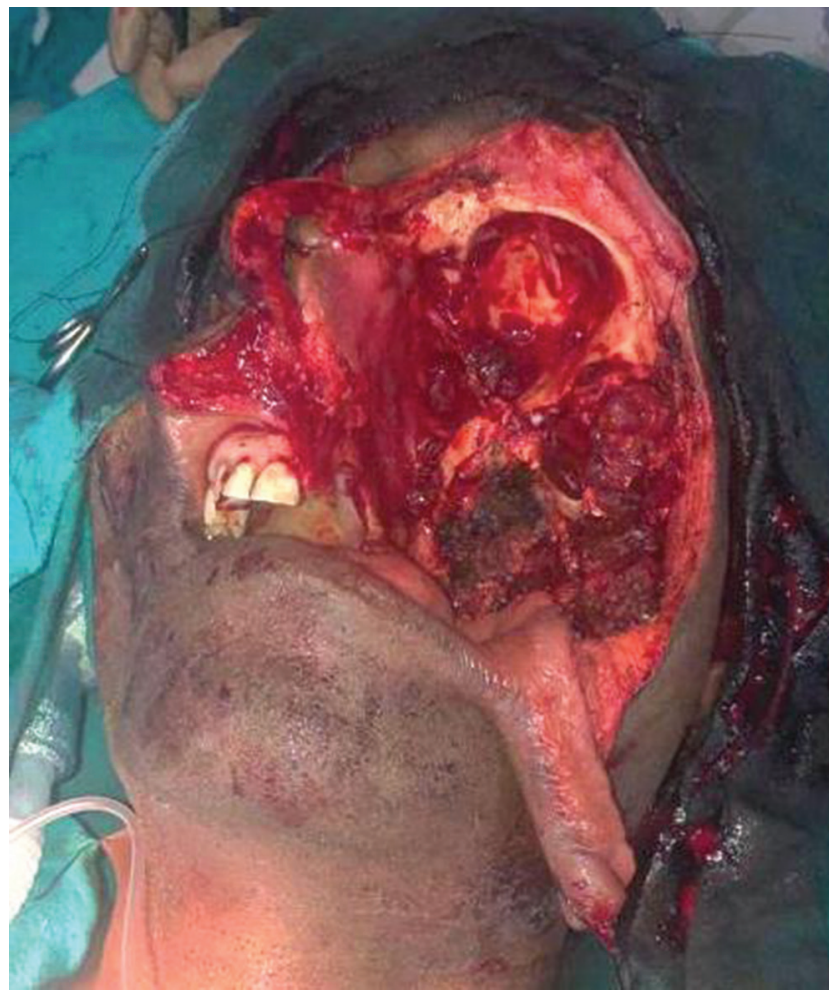

Fig. 6 Postoperative surgical defect. 
floor of the maxillary sinus and across the antral roof, on coronal view, and divided into infrastructure, mesostructure, and suprastructure. Tumors extending into the suprastructure also often require an orbital exenteration. Sisson ${ }^{12}$ et al suggested inclusion of carcinoma of the antrum into the TNM classification. $\mathrm{T} 1$ tumors are confined to the mucosa, T2 lesions are associated with osseous erosion or destruction, and T3 and T4 tumors extend outside the sinonasal cavity into the masticator space, cheek, adjacent paranasal sinuses, orbital apex, base of skull, nasopharynx, or intracranially. ${ }^{13}$ Contrast-enhanced imaging is essential to assess the extent of local disease, and

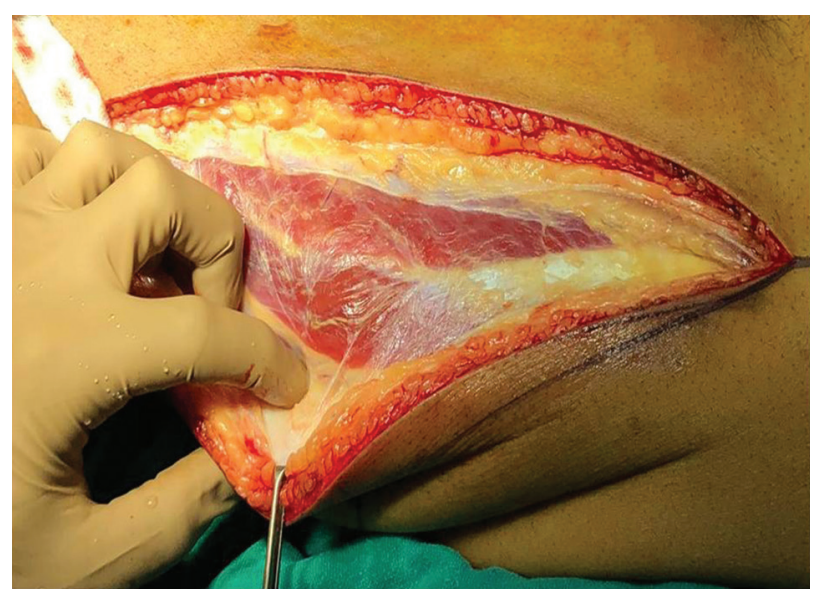

Fig. 7 Harvesting of anterolateral thigh flap.

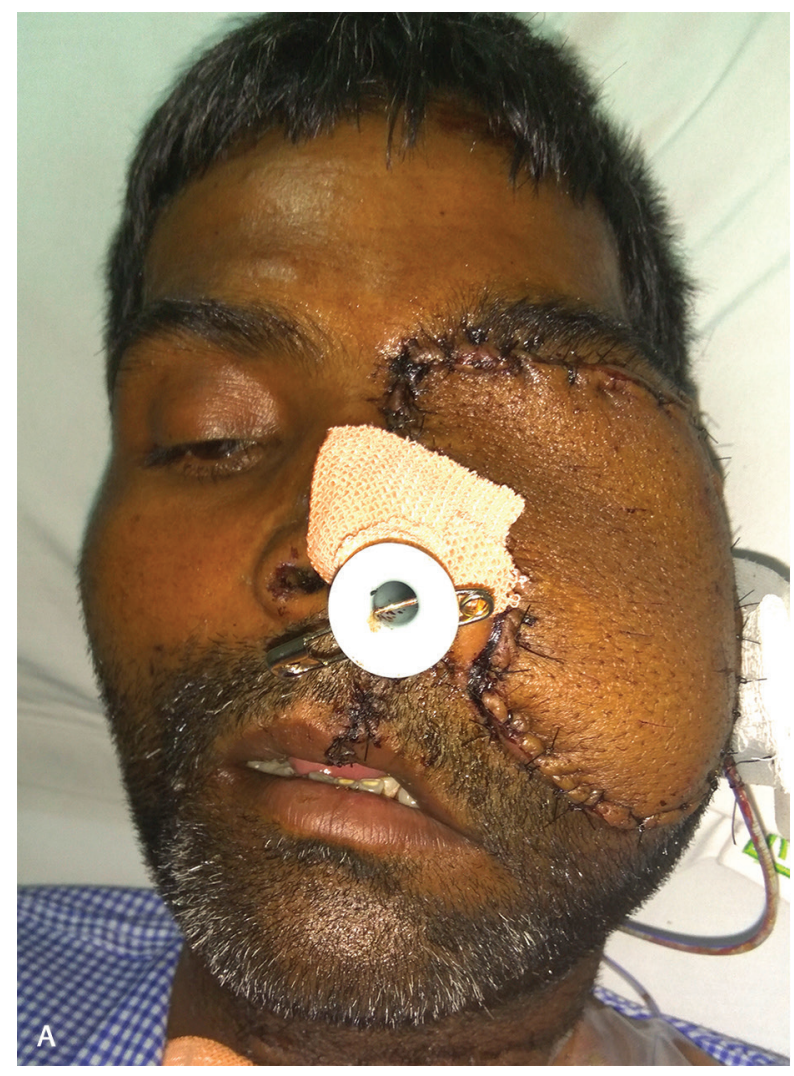

Fig. 8 (A, B) Postoperative extraoral view showing viable flap. the presence of perineural spread and intracranial extension. Potential areas of tumor extension that must be assessed in all patients with sinonasal malignancies include intracranial spread (the anterior and middle cranial fossa), the palate, the orbits, the pterigopalatine fossa, and the skull base. ${ }^{14}$

Spreading of sinonasal malignancies usually takes place directly or by perineural extension. Prognosis and designing of the surgical management depend on superior and posterior boundaries of the maxillary sinuses. If there is posteriorly direct extension of tumor into the orbit, or spread to the intracranial compartment via the ethmoid air cells, then it is difficult to obtain tumor-free surgical margins. Medially, extension to the nasal cavity and inferiorly to the alveolus leads to less problematic en bloc resection..$^{15}$ The lamina papyracea, lateral wall of the ethmoid air cells when violated, may result in intraorbital spread that requires orbital exenteration. Duke-Elder ${ }^{16}$ states that carcinoma of the maxillary sinus is the commonest malignant disease of any of the sinuses to invade the orbit. Iannetti ${ }^{17}$ et al identified three stages of orbital invasion: Grade I, erosion or destruction of the medial orbital wall; Grade II, extraconal invasion of the periorbital fat; Grade III, invasion of the medial rectus muscle, optic nerve, ocular bulb, or the skin overlying the eyelid. They propose that only grade III orbital invasion warrants orbital clearance or exenteration. Regional lymph node metastases from sinonasal malignancies are not so common, but when present, usually indicate tumor extension outside of the sinonasal cavity and poor prognosis. Lateral retropharyngeal nodes are primary nodal drainage site. Therefore, the upper internal jugular and submandibular nodes are the most common sites for nodal metastases. The above-mentioned case presenting with Grade III stage of orbital invasion presented with the following clinical symptoms: globe displacement, limitation of extraocular movements, inequality of palpebral fissures, and palpable mass overlapping the orbit. Left submandibular lymph nodes were palpable, and Level II and III nodes were also enlarged, with no significant indication of distant metastasis. In our case, TNM staging is cT3N3bM0.

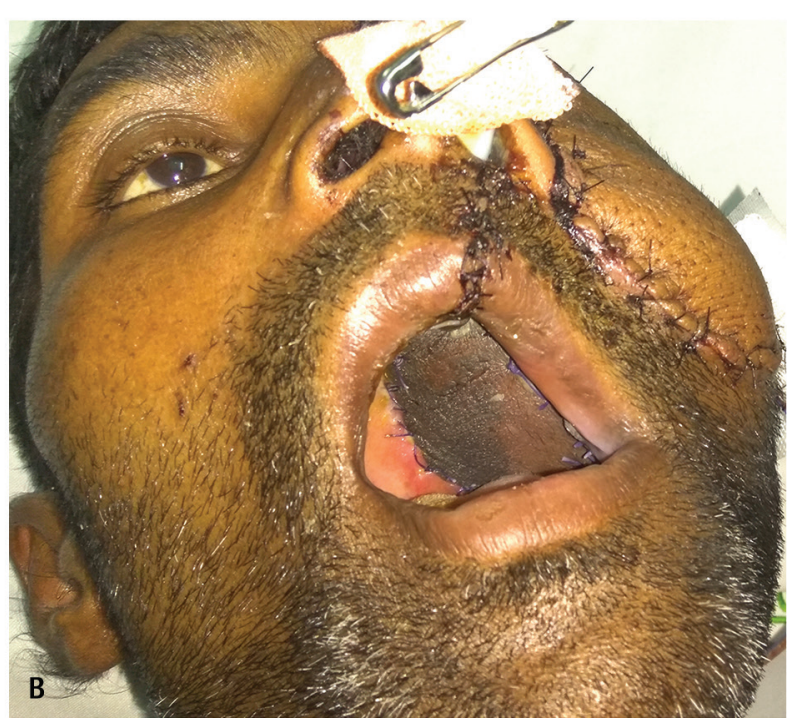


We have performed combined treatment in cases of locally advanced maxillary sinus carcinoma which includes en bloc radical maxillectomy by making the Weber-Ferguson incision with orbital exenteration along with modified neck dissection and reconstruction of defect with flap, followed by radiotherapy. Cordeiro's classification ${ }^{18}$ of maxillary defects provide an idea about extent of maxillectomy procedure. In the above-mentioned case, it was Type IIIb (total maxillectomy with orbital exenteration).

Ketcham et $\mathrm{al}^{19}$ suggested combined intracranial facial approach to overcome the difficulty in the complete extirpation of the cancer. Jesse ${ }^{20}$ suggested radical maxillary resection, followed by radiotherapy, permits definition of the extent of the tumor at the initial surgery for moderately advanced cancer in older people. Yasuo et $\mathrm{al}^{21}$ included chemotherapy in combination with radiation and surgery to overcome the limitation of oncologic excisional surgery. Som et $\mathrm{a}^{22}$ have suggested that orbital preservation is possible by preoperative radiation therapy. However, there is no significant improvement in survival rate by use of planned combination therapy, radiation therapy before or after surgery. Larson et $\mathrm{a}^{23}$ reported indications for orbital exenteration which included invasion of the periorbita, infraorbital nerve, posterior ethmoid sinuses, or orbital apex, and suggested that the survival of patients whose orbit was preserved was not different from those whose orbits were exenterated. In 1988, Perry ${ }^{9}$ et al reported their experience preserving the orbit, even when a sinonasal tract malignancy has invaded the periorbita (localized invasion), and concluded that preservation of the orbit in selected cases did not compromise the cure rate of these patients.

\section{Conclusion}

Carcinoma of the maxillary sinus presents the typical signs, symptoms, and course of the disease. CT and MR imaging play complementary roles in the assessment and staging of these malignancies by determining the presence or absence of extension of disease. Patient survival rate can be increased in patients of maxillary sinus carcinoma by way of a collaboration of oncologist and radio-oncologist. This association includes aggressive surgical therapy by performing en bloc radical maxillectomy with orbital exenteration accompanied by postoperative radiotherapy and chemotherapy.

\section{Conflict of Interest}

None declared.

\section{References}

1 Barnes L, Verbin RS, Gnepp DR, Diseases of the nose, paranasal sinuses, and nasopharynx. In: Barnes L, ed. Surgical Pathology of the Head and Neck. New York: Marcel Dekker; 1985 403-51

2 Chaudhry AP, Gorlin RJ, Mosser DG. Carcinoma of the antrum: a clinical and histopathologic study. Oral Surg Oral Med Oral Pathol 1960;13:269-281
3 Lenworth N. Johnson, Gregory B. Krohel, Elizabeth B. Yeon, Steven M. Sinus tumors invading the orbit. Ophthalmology 1984;91:209-217

4 Keane WM, Atkins JP Jr, Wetmore R, Vidas M. Epidemiology of head and neck cancer. Laryngoscope 1981;91(12):2037-2045

5 Weber AL, Stanton AC. Malignant tumors of the paranasal sinuses: radiologic, clinical, and histopathologic evaluation of 200 cases. Head Neck Surg 1984;6(3):761-776

6 Hesselink JR, Weber AL. Pathways of orbital extension of extraorbital neoplasms. J Comput Assist Tomogr 1982;6(3):593-597

7 Graamans K, Slootweg PJ. Orbital exenteration in surgery of malignant neoplasms of the paranasal sinuses. The value of preoperative computed tomography. Arch Otolaryngol Head Neck Surg 1989;115(8):977-980

8 Wu X, Tang P, Qi Y. Management of the orbital contents in radical surgery for squamous cell carcinoma of the maxillary sinus. Chin Med J (Engl) 1995;108(2):123-125

9 Perry C, Levine PA, Williamson BR, Cantrell RW. Preservation of the eye in paranasal sinus cancer surgery. Arch Otolaryngol Head Neck Surg 1988;114(6):632-634

10 Ohngren LG. Malignant tumors of the maxillo- ethmoid region. Acta Otolaryngol 1933; (19):471-476

11 Lederman M. Tumours of the upper jaw: natural history and treatment. J Laryngol Otol 1970;84(4):369-401

12 Sisson GA, Johnson NE, Amiri CS. Cancer of the maxillary sinus: clinical classification and management. Ann Otol Rhinol Laryngol 1963;72:1050-1059

13 Carinci F, Curioni C, Padula E, Calearo C. Cancer of the nasal cavity and paranasal sinuses: a new staging system. Int J Oral Maxillofac Surg 1996;25(1):34-39

14 Phillips CD, Futterer SF, Lipper MH, Levine PA. Sinonasal undifferentiated carcinoma: CT and MR imaging of an uncommon neoplasm of the nasal cavity. Radiology 1997;202(2):477-480

15 Curtin HD, Williams R, Johnson J. CT of perineural tumor extension: pterygopalatine fossa. AJNR Am J Neuroradiol 1984;5:731-737

16 Duke-Elder S. Textbook of Ophthalmology. St. Louis, Mosby; 1952

17 Iannetti G, Valentini V, Rinna C, Ventucci E, Marianetti TM. Ethmoido-orbital tumors: our experience. J Craniofac Surg 2005;16(6):1085-1091

18 Cordeiro PG, Santamaria E. A classification system and algorithm for reconstruction of maxillectomy and midfacial defects. Plast Reconstr Surg 2000;105(7):2331-2346, discussion 2347-2348

19 Ketcham AS, Wilkins RH, Vanburen JM, Smith RR. A combined intracranial facial approach to the paranasal sinuses. Am J Surg 1963;106:698-703

20 Jesse RH. Paranasal sinuses and nasal cavity. In: MacComb WS, Fletcher GS, eds. Cancer of Head and Neck. Baltimore, Williams \& Wilkins; 1967:329-356

21 Sato Y, Morita M, Takahashi HO, Watanabe N, Kirikae I. Combined surgery, radiotherapy, and regional chemotherapy in carcinoma of the paranasal sinuses. Cancer 1970;25(3):571-579

22 Som ML. Surgical management of carcinoma of the maxilla. Arch Otolaryngol 1974;99(4):270-273

23 Larson DL, Christ JE, Jesse RH. Preservation of the orbital contents in cancer of the maxillary sinus. Arch Otolaryngol 1982;108(6):370-372 